\title{
The Development and Process Evaluation of a 3-Day Acceptance and Commitment Therapy Group Program for Adolescent Cancer Survivors
}

\author{
Kristina Clarke ${ }^{1}$ Pandora Patterson ${ }^{1,2}$ (D) Fiona E. J. McDonald ${ }^{1,2}$ (D) \\ Claire E. Wakefield ${ }^{3,4}$ (D) Ursula Sansom-Daly ${ }^{3,4}$ (D) Brad Zebrack $^{5}$
}

Published online: 28 August 2020

(C) The Author(s) 2020

\begin{abstract}
Background Adolescents diagnosed with cancer experience unique psychosocial concerns that persist beyond treatment completion into longer-term survivorship. Camp-based, group Acceptance and Commitment Therapy (ACT) programs are a potential model for providing evidence-informed psychological and peer support to adolescent cancer survivors.

Objective This paper describes the development and exploration of the feasibility and acceptability of such a program, Places You'll Go. This manualised program incorporates five 90-min group ACT sessions within a 3-day camp, teaching ACT strategies in the context of psychosocial impacts of cancer.

Method Eight facilitators and twenty-eight Australian adolescent cancer survivors (68\% female; age range $12-17$ years, $M=15.4$ years) participated in the program and evaluation. Feasibility was assessed using facilitator-reported session duration, attendance, quality and content fidelity; facilitators also completed interviews after program completion. Young people completed surveys on program acceptability at the end of each session and at program completion.

Results All planned sessions were delivered, with 97\% attendance and high fidelity in manualised program delivery. All young people were mostly or very satisfied and would recommend the program to another cancer survivor. Opportunities for peer connection and skill development contributed to perceived program acceptability.

Conclusions The Places You'll Go program was acceptable and feasible to deliver. It is a promising community-based model for promoting peer support and well-being in adolescent cancer survivors, indicating the potential of ACT-based approaches for this population. Further work is underway to evaluate whether the program improves psychosocial wellbeing among participants, and if this is linked to the therapeutic mechanisms underpinning ACT.
\end{abstract}

Keywords Acceptance and commitment therapy - Adolescent and young adult · Cancer · Cancer survivorship $\cdot$ Intervention

Pandora Patterson

researchteam@canteen.org.au

Extended author information available on the last page of the article 


\section{Introduction}

The rapid psychosocial development experienced in adolescence, including changes in identity, independence, and relationships (Steinberg and Morris 2001), is disrupted by a cancer experience. Globally, good survival rates for children and adolescents diagnosed with cancer (GBD 2017 Childhood Cancer Collaborators 2019) mean that there is a growing population of young people at risk for experiencing ongoing impacts of a cancer diagnosis and its treatment. In Australia, approximately 1000 children and adolescents (0-19 years) are diagnosed with cancer each year, with survival rates at $84 \%$ for children (0-14 years) and $89 \%$ for young people (15-24 years; AIHW 2017).

Although many adolescent cancer survivors ${ }^{1}$ adjust well to their cancer experience (Gunst et al. 2016; Niepage et al. 2018; Yi et al. 2015), a significant minority report psychosocial challenges after completing cancer treatment. Psychological impacts include clinically-significant distress, post-traumatic stress, and anxiety (Friend et al. 2018; Rourke et al. 2015); fear of cancer recurrence; perceptions of being different; challenges incorporating the cancer experience into their identity; and concerns about cancer-related disclosure with peers, friends and partners (Janin et al. 2018; Kim et al. 2016; McDonnell et al. 2017; Pini et al. 2019). Social impacts include social isolation and altered interpersonal relationships; need for peer support from other cancer survivors (Barnett et al. 2016); and disrupted education, work, and finances (Fardell et al. 2018).

As adolescent cancer survivors face the daunting task of managing "normal" life alongside the impacts of their cancer experience (Woodgate 2005), access to appropriate professional services and support to address unmet needs is needed (Baird et al. 2019; Barnett et al. 2016; Tsangaris et al. 2014; Wakefield et al. 2013). However, relatively few evidencebased interventions are available addressing the psychosocial needs of this population: Walker et al.'s (2016) review identified just eleven high quality publications evaluating interventions for 10-30 year olds with cancer, of which few targeted only the adolescent age range and/or reported a specific therapeutic approach. ${ }^{2}$

Those that did use a therapeutic approach included Beaupin et al.'s (2018) meaningcentred psychotherapeutic intervention, where young people (15-24 years) shared photos and narratives with each other in response to ten weekly prompts on an online platform; and Sansom-Daly et al.'s $(2012,2019)$ online, group-based cognitive behavioural therapy intervention where young people aged 15-25 years were taught skills over six weeks in the context of early survivorship concerns. In these cases, only data on the feasibility and acceptability of these interventions has thus far been presented. Whilst this preliminary evidence suggests that both approaches have appeal to young cancer survivors, there is another therapeutic approach which shows promise for this population.

\section{Acceptance and Commitment Therapy (ACT)}

Increasingly, the field of psychology has seen a shift from typical behavioural only paradigms (first wave therapies) to cognitive behavioural paradigms (second wave) to new third

\footnotetext{
${ }^{1}$ Used here to refer to adolescent-aged young people (12-17 years) diagnosed with cancer during childhood ( $0-11$ years) or adolescence (12-17 years).

${ }^{2}$ The majority were educational and/or exercise interventions, taught general coping skills, or involved non-specific individual or group sessions.
} 
wave therapies that include Acceptance and Commitment Therapy (ACT). ACT incorporates behavioural change techniques but focuses on changing the context and therefore the individual's relationship with distressing thoughts and feelings. Third wave paradigms identify and emphasise strengths that facilitate growth thereby reducing distress, in contrast to therapies that focus on eliminating "problems" (Hayes et al. 1999).

ACT aims to help people build psychological flexibility, or the ability to willingly experience emotions and "let go" of ineffective struggles with unwanted thoughts or feelings in the service of engaging in values-consistent behaviour (Hayes et al. 2006). Psychological flexibility is developed through the ACT processes of acceptance-based coping, defusion and mindfulness, and commitment to values-consistent behaviour. These components of psychological flexibility have been linked with well-being in young people. For example, dispositional mindfulness has been linked to lower distress in adolescents with cancer (Patterson and McDonald 2015). Acceptance has been linked to increased positive affect and decreased sadness in Australian adolescents (Ciarrochi et al. 2011) and lowered distress in adolescents with severe chronic pain (Pielech et al. 2017). Longitudinal research suggests that both awareness and acceptance are critical components of positive adolescent development (Ciarrochi et al. 2011, 2008). Moreover, ACT-based interventions have a demonstrated ability to improve engagement in these underlying therapeutic processes, including psychological flexibility, mindfulness, and values-based living (Halliburton and Cooper 2015).

ACT has recently gained popularity as a treatment approach to address psychosocial stressors for a broad range of concerns. Randomised controlled trials show ACT to be effective across different populations for various mental health and medical concerns (A-Tjak et al. 2015). In particular, an emerging body of evidence has highlighted ACT as a feasible and acceptable approach for adolescents experiencing conditions such as depression, post-traumatic stress disorder, and chronic pain (Ernst and Mellon 2016; Halliburton and Cooper 2015), and with adolescents with chronic illness (Ernst and Mellon 2016). ACT has also been reported to have potential for use in interventions supporting young people impacted by a familial cancer diagnosis or bereavement (Ing et al. 2019; Patterson et al. 2015).

Multiple features of ACT make it an appropriate therapeutic approach for adolescent cancer survivors. This includes its transdiagnostic approach that can be flexibly applied across a range of concerns for young people (Coyne et al. 2011a, b); a focus on experiential learning that may be engaging for adolescents (Swain et al. 2015); an emphasis on changing one's relationship with one's thoughts, while acknowledging realistic fears of cancer recurrence (Jones et al. 2013); a focus on values-based living; and flexible self-view that could help adolescents process changes to their identity and goals after cancer (Halliburton and Cooper 2015).

The ability to deliver ACT in a peer group format is particularly important for the adolescent cancer survivor population who may experience isolation resulting from reduced opportunities for meaningful social connection with their friends and social networks (Janin et al. 2018; Kim et al. 2016). Connections with other cancer survivors can reduce social isolation; promote a sense of community or belonging; and normalise and validate psychosocial impacts through shared information and experience (Barnett et al. 2016; Niepage et al. 2018). As has been evidenced for young people with mental health concerns (Halliburton and Cooper 2015; Livheim et al. 2014), group-based ACT approaches may offer additional or enhanced therapeutic benefits for adolescents with cancer. 


\section{The Current Study}

This paper outlines the development and process evaluation of Places You'll Go, a new camp-based ACT program for adolescent cancer survivors delivered by Canteen. ${ }^{3}$ The study aimed to develop this novel intervention and explore its feasibility and acceptability to young people and facilitators. While they do not determine the psychosocial impacts of program participation, process evaluations are a necessary first step for novel interventions to ensure adequate description of the intervention and its components; determine viability and acceptability; and to identify barriers and enablers that will inform optimal future delivery (Chung et al. 2015; Mendel et al. 2008). More broadly, the paper's contributions also indicate the feasibility and value of (i) the therapeutic approach used (ACT) for this population, and (ii) community-based support for adolescent cancer survivors. Based on previous literature, it was hypothesised that this ACT-based group program would be perceived as acceptable and useful to adolescent cancer survivors. This paper additionally lays the foundation for subsequent work evaluating the impacts of the Places You'll Go program on participants' psychosocial wellbeing.

\section{Method}

\section{The Places You'll Go Program}

The Places You'll Go program was developed using a multi-stage process: (1) scoping of the existing literature on the psychosocial impacts of cancer for adolescents and currently available psychosocial interventions; (2) a content review of Canteen's existing services for cancer patients and survivors and existing camp-based programs; and (3) consultation with Canteen staff and young people about preferred program content and format. The program development team comprised both research and clinical professionals with expertise in adolescent and young adult psycho-oncology, including those with lived experiences of having cancer as a young person.

Five topics relevant to adolescent cancer survivors were identified as priorities for discussion during the program: peer connection and sense of community; identity and meaning-making; distress associated with uncertainty and cancer-related worries (e.g., fear of cancer recurrence); cancer-related disclosure; and goal-setting and valued activity participation. ACT concepts were mapped onto these topics, and program activities developed to teach specific ACT strategies relevant to coping with each survivorship concern (e.g., acceptance and defusion as a mechanism for managing uncertainty and fear of recurrence). The pilot program reported on in this paper was a 3-day, 2-night camp, with the core therapeutic component a series of five 90-minute psychosocial sessions. Peer connection and support opportunities were emphasised throughout the program and recreational activities were interspersed with therapeutic sessions. ACT principles (e.g. mindful presence, acceptance of emotional experiences) were also reflected and reinforced throughout the program. A program manual was developed to help facilitators prepare for and deliver

\footnotetext{
${ }^{3}$ Canteen is the national organisation for young people living with cancer. Canteen provides psychosocial support to 12 to 25 year olds who have been diagnosed with cancer, have a close family member who has been diagnosed with cancer, or have had a close family member die due to cancer. For more information see www.canteen.org.au.
} 
Table 1 Psychosocial session content on the Places You'll Go program

\begin{tabular}{|c|c|}
\hline Session & Content \\
\hline $\begin{array}{l}\text { Session One: Introduction to your group and your } \\
\text { values }\end{array}$ & $\begin{array}{l}\text { Welcome to the group and introduction to the } \\
\text { program and other group members. Develop- } \\
\text { ment of a group contract for working together in } \\
\text { session groups. 'Getting to know you game' with } \\
\text { question and conversation prompts to facilitate } \\
\text { group cohesion and identification of shared experi- } \\
\text { ences. Psychoeducation on values and goals, with } \\
\text { opportunities to identify own values and discuss } \\
\text { how people show these values. Introduction to the } \\
\text { concept of mindfulness and learning to be aware of } \\
\text { what is happening in the present moment. Mindful } \\
\text { breathing practice. Debrief to assist people to pro- } \\
\text { cess group experience and shared connections. }\end{array}$ \\
\hline
\end{tabular}

Session Two: Exploring your identity and meaningmaking after cancer

Session Three: Accepting uncomfortable thoughts and feelings

Session Four: Sharing your cancer story

Session Five: Committing to the places you'll go next!
Develop understanding of identity and the impact of adolescence and one's cancer experience on one's identity. Craft-based activity to explore different parts of self, including the 'visible'/external and 'invisible'/internal. Video-facilitator discussion of changes in identity and values after cancer to introduce concepts of flexible self-view and meaningmaking. Mindful listening practice.

Psychoeducation about acceptance and the idea that getting caught up in unwanted thoughts and feelings can stop one from doing the things they care about. Experiential craft-based introduction to defusion. Learning to have a different relationship with one's thoughts and introduction of a strategy to detach from an unwanted thought or feeling. Mindful colouring practice.

Explores the group's experience with sharing their cancer story with peers, friends and family and the values that might influence wanting to do this. Teaches a method for developing an action plan for how they could share their cancer story with a person of their choice. Psychoeducation on the concept of committed action, with discussion of obstacles that might come up when sharing their cancer story and strategies for accepting or working through obstacles.

Identifying values and goals they would like to focus on after the program. Psychoeducation on writing S.M.A.R.T. goals. Development of a committed action plan to help them take values-based action after the program and head in the direction they want to go in life, whilst still allowing the experience of unwanted thoughts and feelings. Letterwriting activity to a hypothetical cancer patient to explore the changes in identity and coping strategies they would like to share with someone else. An opportunity to reflect on what has been learnt in group sessions and celebrate group work. 
the five psychosocial sessions; see Table 1 for an outline of content covered during these sessions.

PP, FM and KC are employees of Canteen, which owns the intellectual property rights to the Places You'll Go program.

\section{Facilitators}

Facilitators were psychosocial support workers with Canteen with expertise in working with adolescents impacted by cancer. All had previous, general ACT training as part of their work, and were therefore able to support and reinforce the ACT approach in both the therapeutic sessions and in the broader program. They also undertook specific training to deliver the Places You'll Go program, as is briefly overviewed in the intervention delivery section below.

\section{Participants}

The Places You'll Go program was advertised to Canteen members, as well as through organisational networks. Program participation was open to all adolescents (aged 12-17 years) who had been diagnosed with cancer, and who had completed curative cancer treatment or were receiving long-term or maintenance therapy. No other inclusion or exclusion criteria (including any related to participants' baseline levels of unmet needs or psychosocial wellbeing) were specified. All young people who attended the Places You'll Go program were invited to participate in the evaluation. All facilitators who delivered manualised session content were also invited to participate.

\section{Procedure}

\section{Recruitment}

Participant information sheets and consent forms were sent to young people and facilitators at the time they were confirmed as attending the program; parental consent forms were used for young people aged less than 16 years old. All participants were assured that their involvement in the evaluation was voluntary and would not affect their attendance on the Places You'll Go program. Consent for demographic data collection and use is provided to Canteen at commencement of service use. Study approval was sought from the Human Research Ethics Committee at the University of Sydney (protocol number 2017/013).

\section{Intervention Delivery}

Simultaneous sessions were delivered to four groups of seven young people, with two lead psychosocial facilitators per group. These sessions were held in quiet indoor settings at the program location (e.g. meeting rooms of a conference centre). There were 20 planned program sessions (five sessions for each of the four groups) of $90 \mathrm{~min}$ each. Each session included five to six planned activities (five for sessions 2, 3, and 4; six for sessions 1 and 5). Prior to program delivery, facilitators attended a two-day training workshop on the program sessions and activities; this was delivered by Canteen staff with expertise in 
psychology, ACT, supporting adolescent cancer survivors, group program delivery, and program evaluation. The program reported on in this paper was run in February 2017.

\section{Evaluation Design}

Data on program feasibility and acceptability were collected from young people and facilitators. Both groups completed brief questionnaires at the end of each session. Post-program questionnaires were completed by young people on the final day of the program; interviews were completed with facilitators by phone, one month after program end.

\section{Measures}

\section{Demographics}

Demographics for young people consisted of date of birth, diagnosis, and treatment completion; type of cancer diagnosis; and gender identity. Information on facilitators' professional background was collected.

\section{Program Feasibility}

Program feasibility was measured using author-developed implementation fidelity questionnaires. Facilitators recorded young people's attendance at sessions; session start and end time (session duration); self-perceived session delivery competence (session quality; scale from $0=$ not competent to $4=$ extremely competent); and whether activities were delivered as intended (session content fidelity). To assess content fidelity, activities were recorded either as delivered as per the manual, adapted (i.e., partially delivered or modified), or not delivered. Facilitator questionnaires had space to provide details of any adaptations, along with general session feedback.

\section{Program Acceptability}

Program acceptability was measured using author-developed satisfaction questionnaires for facilitators and young people, and post-program interviews with facilitators. Young people rated their agreement that each session was helpful, meaningful and relevant, and interesting to them on a 7 -point scale $(1=$ strongly disagree to $7=$ strongly agree). Space was provided for general comments. At program completion, young people rated program satisfaction on a 4 -point scale $(1=$ not at all satisfied to $4=$ very satisfied); indicated whether they would recommend the program to another adolescent cancer survivor (yes/no); and answered open-ended questions on the most and least useful and liked elements of the program, as well as areas for improvement. At completion of each session, facilitators rated their agreement that adolescents were engaged in the session on a 7 -point scale $(1=$ strongly disagree to $7=$ strongly agree $)$. Postprogram facilitator interviews explored program acceptability, barriers and enablers to session delivery. Example questions included "What are your impressions of the Places You'll Go psychosocial [facilitator manual/session format]?", "What worked well?", and "What would you change?". 
Table 2 Participant demographics

\begin{tabular}{ll}
\hline Characteristic & Program participants $(n=28)$ \\
\hline Age at program start in years; mean, $(S D)$, range & $15.4,(1.6), 13.1-17.8$ \\
Gender (\%) & 68 \\
Female & 32 \\
Male & \\
Cancer type (\%) & 29 \\
Brain \& Central Nervous System & 14 \\
Bone \& Articular Cartilage & 4 \\
Liver & 21 \\
Leukemia & 21 \\
Lymphoma & 7 \\
Connective \& other soft tissue & 4 \\
Non-cancer diagnosis* & \\
Age at diagnosis (\%) & 18 \\
Infancy to early childhood (0-5 years) & 29 \\
Middle childhood (6-11 years) & 53 \\
Adolescence (12-17 years) & $4.8,(4.0), 0.7-13.5$ \\
Time since diagnosis in years; mean, $(S D)$, range & $3.8,(3.7), 0.1-13.1$ \\
Time since curative treatment in years; mean, $(S D)$, range & \\
\hline
\end{tabular}

*Young people without a cancer diagnosis were eligible for the program if they had been treated using similar treatment approaches or protocols (e.g., chemotherapy)

\section{Data Analysis}

Quantitative data were analysed using descriptive statistics to determine whether the intervention was feasible and acceptable. Individual group scores were calculated for feasibility data (session attendance, duration, quality, and content fidelity) by collapsing responses from the two facilitators in each of the four groups. Where session scores are reported, this indicates ratings collapsed across the four groups. Where program scores are reported, this indicates ratings collapsed across groups and the five sessions.

Written responses to open-ended questions and interviews were transcribed by two research assistants with no prior project involvement. As participants' and facilitators' responses typically took the form of brief feedback on the program, a formal approach to thematic analysis was considered inappropriate. Instead, responses to open-ended questions and interviews were reviewed by the authors to identify topic areas mentioned by multiple facilitators and young people. Example comments are included in this paper to illustrate selected key topics. 


\section{Results}

\section{Participants}

The Places You'll Go program was attended by twenty-eight young people, all of whom provided feedback on the program's acceptability (68\% female; mean age $=15.4$ years, range 13.2 to 17.8 years). Full participant demographics for young people can be seen in Table 2. Eight facilitators participated in psychosocial session delivery and provided feedback on program feasibility, with six facilitators completing interviews. Facilitators' professional backgrounds included social work, psychology, education, nursing, and occupational therapy.

\section{Program Feasibility}

Descriptive statistics for feasibility and acceptability of individual sessions and the program can be seen in Table 3 .

Facilitator report indicated that all planned sessions were delivered, with $97 \%$ attendance by young people (range 96-100\% across sessions) and with a mean session duration of 87 min (range 81-91 min across sessions). As an indicator of session quality, across program sessions $39 \%$ of facilitators gave a self-perceived session delivery competence rating of very or extremely competent (range $25-50 \%$ across sessions). Content fidelity measures indicated $70 \%$ of activities were delivered as outlined in the manual (range 55-85\% across sessions); $25 \%$ were delivered partially or with some modifications (range 13-35\% across sessions); and 5\% of activities were not delivered (range 0-10\% across sessions).

Session modifications described by facilitators included adding a warm-up/icebreaker activity to the start of a session; extending or shortening activity length from the timing suggested in the manual; modifying the suggested group structure (e.g., running activities in pairs or smaller groups); providing additional metaphors or examples for ACT processes and strategies; using more concrete or abstract examples to suit the age and developmental stage of group members; incorporating additional discussion on topics raised by young people; and choosing not to use available resources to focus discussion.

Facilitator comments on questionnaires and in interviews reflected three common reasons for modification or partial delivery of activities. First, facilitators described needing to adapt activities to suit the needs of the group; for example, based on age at diagnosis, current age, or presenting concerns.

We split groups into older and younger and then, recently survived and longer-term survivorship,, so each group I guess probably approached the material differently... [F3; Interview].

Second, some facilitators reported that delivery of some activities took longer than expected, meaning later activities were shortened or partially delivered.

The video/script generated a lot of rich discussion, which then impacted the time available for mindfulness.[F7; Session 2].

Third, facilitators reported that environmental challenges prevented activity delivery; for example, a loud thunderstorm that occurred during Session 2 prevented young people from being able to hear and participate in a video-based activity. 


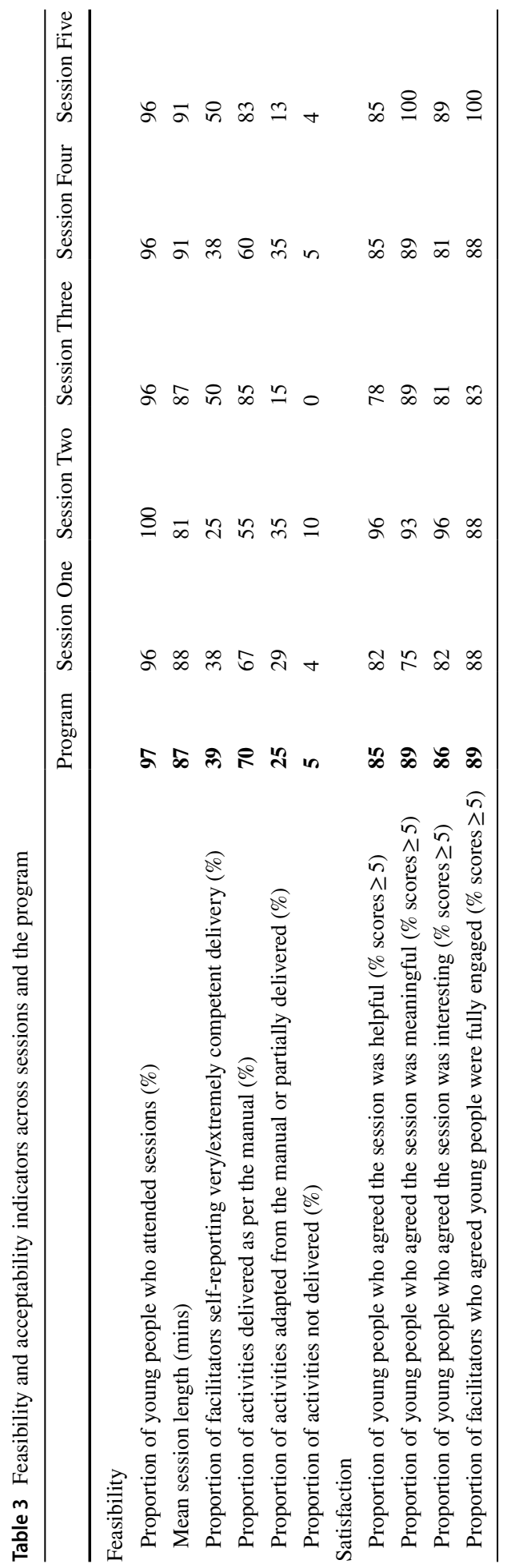


Storm too loud, video unable to be played or heard. Attempted to plug into audiovisual system. Could not play.[F1, Session 2].

Facilitators' comments on reasons for low session delivery self-competence ratings indicated that they did not feel able to spend as much time preparing for sessions prior to the program as they would have liked.

I think really we just didn't have enough time to do all of the activities [before the program] and you could really tell on program the ones that we had practiced and the ones that we hadn't.[F1, Interview].

Program characteristics perceived by facilitators as enabling competent session delivery were the availability of prepared resources and access to the support and knowledge of other program staff before and during the program.

So the team had put together all of the resources, really clearly labelled... from the facilitator point of view not having to worry about that was absolutely, just a dream... [F7, Interview].

I found it very helpful to have the group, the facilitators, come together for at least two pre-weekend orientation meetings; that just really helped me to get an understanding for what the expectations were going to be for me as a facilitator over the weekend.[F8, Interview].

\section{Program Acceptability}

All program participants (100\%) reported being mostly or very satisfied with the overall Places You'll Go program and that they would recommend the program to another adolescent cancer survivor. Regarding acceptability of session content, $85 \%$ of participants agreed that sessions were helpful (range 78-96\% across sessions); 89\% agreed that sessions were meaningful and relevant (range $75-100 \%$ across sessions); and $86 \%$ agreed that sessions were interesting (range 81-96\% across sessions). Eighty-nine per cent of facilitators agreed that participants were fully engaged in sessions (range $83-100 \%$ across sessions).

Comments on end-of-session and end-of-program acceptability measures reflected three program elements with which young people were most satisfied. First, young people reported enjoying having opportunities for peer connection and support; "[I liked] meeting a heap of new friends who share the same experience" [YP6, End of Program]; "I would recommend it because it is so great to know that you're not alone and you have lots of support" [YP20, End of Program].

Second, young people reported being satisfied with the relevance of the program content to their survivorship experience; "It was practically based around all of our experience and I was able to share and have people understand" [YP2, End of Program]; "The program was based a lot on what I had gone through which was good" [YP 3, End of Program].

Third, young people reported satisfaction with the opportunities to learn specific new skills they could use after the program; "[Most useful] was when we did our sessions and talked about some strategies to help us cope with negative feelings and thoughts" [YP13, End of Program]; "The mindfulness exercises were most useful to me" [YP7, End of Program].

The importance of these elements was emphasised by the few young people who reported lower satisfaction when these elements were not present. For example, if they did not relate to other young people on the program ("Personally, I didn't meet anyone with 
a similar experience to me" [YP18, End of Program]) or if they did not relate to program topics ("I don't get many uncomfortable thoughts and I'm comfortable telling people I had cancer" [YP12, End of Program]).

For facilitators, shared peer connection and relevance to survivorship experience were also described as key strengths of the program.

I think that an obvious strength was the fact that we had a group of patient members together on a camp; time and time again just the young people kept saying "this is just so great," that they felt included, that they were with who they really identified as their peers.[F4, Interview].

... the flexibility within the program to allow the young people to talk about what they need to talk about and process some of the issues that they are or will experience as survivors was an important thing to be able to have... [F7, Interview].

The complexity of the ACT strategies for some young people to understand decreased the perceived acceptability of the program for facilitators.

Well I think it was hard for them to understand...the willingness and acceptance stuff. Yeah. That session was, I don't doubt that it was helpful, but... I think that you need to explain it a number of times at a number of different timepoints for people to really get it.[F7, Interview].

\section{Discussion}

A novel, camp-based ACT group intervention was delivered to 28 adolescent cancer survivors. Results indicate that the program was feasible for facilitators to deliver and highly acceptable to young people and facilitators.

Facilitators reported high adherence to the program manual and its components, providing preliminary evidence that group ACT sessions can be delivered on a camp-based program with adolescents. Across the program, facilitators reported that $70 \%$ of activities were delivered as per the manual, with a further $25 \%$ adapted for the group or situation. This is consistent with previous work, with a review of fidelity in prevention and health promotion programs for children and adolescents finding that few studies achieve fidelity higher than $80 \%$, and that positive outcomes can be achieved with $60 \%$ fidelity (Durlak and DuPre 2008).

Findings from implementation science research have pointed to the need to allow for flexibility and adaptation in manualised program delivery. These previous studies recommend tailoring programs to the group's needs, with an expected positive contribution of tailored adaptation on outcomes (Breitenstein et al. 2010; Durlak and DuPre 2008; Kendall et al. 2008; Mendel et al. 2008). A degree of flexibility was thus built into the facilitator manual for this program. Qualitative feedback from facilitators reflected that adaptations were made to suit group needs; for example, moving from concrete to abstract delivery, or vice versa, based on participant age or developmental stage. However, it is possible that the need for adaptations contributed to the low proportion of facilitators self-reporting feeling very or extremely competent in session delivery.

Interestingly, facilitators' relative lack of confidence in delivering the second session may not necessarily compromise participants' experiences or program benefits. For example, facilitators' self-reported competence and adherence to the program manual were 
lowest for session 2, yet adolescents reported the greatest satisfaction with this session (Table 3). Here, open-ended responses indicated that this may be due to the rich discussions stimulated by the video and script prompt; facilitators' decisions to extend these conversations may have meant adapting or condensing the remaining activities in the session, but the overall effect seems to have enhanced therapeutic benefits for participants.

Future program development and evaluation should consider the amount and types of adaptation that can be made whilst still reflecting program adherence (Perez et al. 2016), as well as the training needed to assist facilitators to feel competent to creatively deliver the program according to clinical need. For example, pre-program training could explicitly address needs and strategies for adapting the program in response to situational demands, which may improve facilitators' self-efficacy to make these changes; providing brief, aggregated feedback from participants' evaluations may also help to reassure facilitators of their abilities, given adolescents' high satisfaction with sessions. Additionally, anticipating common practical barriers to program fidelity (e.g. environmental impacts on use of technology; time constraints) may allow for planning ahead to prevent or better adapt to these challenges when they arise, potentially minimising their impact.

Young people reported high program acceptability, with every participant indicating satisfaction with the program and that they would recommend it to another adolescent cancer survivor. Participants identified key strengths as opportunities for peer support and connection; relevance to the survivorship experience; and opportunities to develop coping skills. The importance of peer support opportunities and connection to cancer experience was also endorsed by facilitators.

Providing camp-based opportunities to connect with other young people with a similar experience addresses an identified unmet need for social support in adolescent cancer survivors (Martins et al. 2017; Penn and Kuperberg 2018). This need may be particularly relevant for the geographically dispersed population of young people in Australia who may have few opportunities to meet other cancer survivors in person. Sessions provided opportunities to receive peer support in specific areas of relevance to adolescent cancer survivors; this peer support element was frequently described as contributing to session ratings of helpfulness and interest. While peer support is not unique to the Places You'll Go program, it lends itself particularly well to combination with ACT approaches. Targeted peer group discussions can help to validate and normalise one's cancer experience, which may be particularly important in helping adolescents to accept the situation and their emotional response. The opportunity to learn from others about how they apply coping strategies and model psychological flexibility also offers short- and long-term benefits to well-being (Martins et al. 2017; Song et al. 2012). The impact of peer support opportunities on wellbeing will be examined in a future outcome evaluation of the program.

Program content was carefully selected to target key areas of concern for adolescent cancer survivors and to address unmet needs for information about strategies for coping with the psychosocial impacts of cancer (Barnett et al. 2016; Rourke et al. 2015). The relevance of selected content was supported by young people and facilitators, alongside perceived satisfaction with opportunities to develop skills for use after the program. Young people identified individual ACT strategies that they felt were the most personally relevant or helpful (e.g., exploring values, coping with uncomfortable thoughts and feelings, mindfulness, and committed action), indicating that ACT strategies are not only acceptable but useful to program participants. This is consistent with previous research indicating the applicability of ACT for young people due to its transdiagnostic approach, focus on experiential learning and strategies, and use of acceptance-based coping strategies rather than challenging of realistic thoughts (Coyne et al. 2011a, b; Halliburton and Cooper 2015). 


\section{Psychosocial Implications and Next Steps}

This study provides preliminary evidence for the feasibility and acceptability of this campbased program as a peer support and therapeutic ACT program for adolescent cancer survivors. This is likely in part due to the embedding of the intervention within the Canteen organisational context, facilitating the recruitment of adolescents to the program and allowing them to benefit from the expertise of staff who facilitated sessions. However, the manualisation of Places You'll Go allows for its replication beyond this context with high fidelity, with the implication that these iterations of the program will be perceived as similarly acceptable and satisfying to participating adolescents.

Collecting information about the process of delivering this program was a necessary first step for evaluating this novel approach to psychosocial intervention. The associations between participant characteristics, intervention characteristics, implementation fidelity, and outcomes should be examined in future effectiveness studies (Cross et al. 2015; Dunst et al. 2013). The small sample size, driven by the relatively small number of adolescent cancer survivors in Australia, meant that this pilot study was underpowered to investigate these associations. Ongoing work is currently underway to deliver a revised version the program to a larger sample of adolescent cancer survivors and collect outcome data that will provide information on the program's efficacy. This work utilises validated measures of ACT processes and psychosocial outcomes, allowing us to determine if and which components of ACT contribute to improved participant wellbeing.

Currently, there are limited opportunities for adolescent cancer survivors to access evidence-informed psychosocial interventions. Collaboration between the healthcare system and community support organisations on the provision of routine survivorship care is important, with community-based organisations able to offer complementary access to therapeutic interventions (Baird et al. 2019). This study demonstrates the feasibility and acceptability of delivering a specifically-tailored ACT intervention for adolescent cancer survivors in a community setting, indicating the potential for such programs to address psychosocial and peer support needs that persist beyond treatment completion.

Acknowledgements The authors would like to acknowledge the contribution of the young people, staff and volunteers who participated in this study.

Funding Canteen Australia is a registered charity that receives funding from a variety of government and individual sources. No specific funding was provided to complete this study. Claire Wakefield is supported by a Career Development Fellowship from the National Health and Medical Research Council of Australia (APP1143767). Ursula Sansom-Daly is supported by Early Career Fellowships from the Cancer Institute NSW (14/ECF/1-11) and the National Health and Medical Research Council of Australia (APP1111800).

Data Availability The data that support the findings of this study are not publicly available as they contain potentially identifying and sensitive information. No ethical approval was obtained for the sharing of this dataset with researchers external to this project.

\section{Compliance with Ethical Standards}

Conflict of interest Authors Patterson, McDonald and Clarke are employees of Canteen Australia. Canteen Australia owns the intellectual property rights to the Places You'll Go program described in this paper, including any potential financial benefits that may result from future program dissemination.

Ethical Approval Ethical approval for this study was granted by the University of Sydney Human Research Ethics Committee (2017/013). 
Informed Consent Written informed consent was obtained from facilitators, participants, and participants' parents (where participants were under 16 years) prior to the program.

Open Access This article is licensed under a Creative Commons Attribution 4.0 International License, which permits use, sharing, adaptation, distribution and reproduction in any medium or format, as long as you give appropriate credit to the original author(s) and the source, provide a link to the Creative Commons licence, and indicate if changes were made. The images or other third party material in this article are included in the article's Creative Commons licence, unless indicated otherwise in a credit line to the material. If material is not included in the article's Creative Commons licence and your intended use is not permitted by statutory regulation or exceeds the permitted use, you will need to obtain permission directly from the copyright holder. To view a copy of this licence, visit http://creativecommons.org/licenses/by/4.0/.

\section{References}

A-Tjak, J. G. L., Davis, M. L., Morina, N., Powers, M. B., Smits, J. A. J., \& Emmelkamp, P. M. G. (2015). A meta-analysis of the efficacy of acceptance and commitment therapy for clinically relevant mental and physical health problems. Psychotherapy and Psychosomatics, 84, 30-36.

Australian Institute of Health and Welfare. (2017). Cancer in Australia 2017. Canberra: AIHW.

Baird, H., Patterson, P., Medlow, S., \& Allison, K. R. (2019). Understanding and improving survivorship care for adolescents and young adults with cancer. Journal of Adolescent and Young Adult Oncology. https://doi.org/10.1089/jayao.2019.0031.

Barnett, M., McDonnell, G., DeRosa, A., Schuler, T., Philip, E., Peterson, L., et al. (2016). Psychosocial outcomes and interventions among cancer survivors diagnosed during adolescence and young adulthood (AYA): A systematic review. Journal of Cancer Survivorship, 10(5), 814-831. https://doi. org/10.1007/s11764-016-0527-6.

Beaupin, L. K., Pailler, M. E., Brewer-Spritzer, E., Kishel, E., Grant, P. C., Depner, R. M., et al. (2018). Photographs of meaning: A novel social media intervention for adolescent and young adult cancer patients. Psycho-Oncology, 28(1), 198-200.

Breitenstein, S. M., Gross, D., Garvey, C. A., Hill, C., Fogg, L., \& Resnick, B. (2010). Implementation fidelity in community-based interventions. Research in Nursing and Health, 33(2), 164-173. https:// doi.org/10.1002/nur.20373.

Chung, O. K., Li, H. C., Chiu, S. Y., Ho, K. Y., \& Lopez, V. (2015). Sustainability of an integrated adventure-based training and health education program to enhance quality of life among Chinese childhood cancer survivors: A randomized controlled trial. Cancer Nursing, 38(5), 366-374. https://doi. org/10.1097/NCC.0000000000000211.

Ciarrochi, J., Heaven, P. C. L., \& Supavadeeprasit, S. (2008). The link between emotion identification skills and socio-emotional functioning in early adolescence: A one-year longitudinal study. Journal of Adolescence, 31, 564-581.

Ciarrochi, J., Kashdan, T. B., Leeson, P., Heaven, P., \& Jordan, C. (2011). On being aware and accepting: A one-year longitudinal study into adolescent well-being. Journal of Adolescence, 34(4), 695-703. https ://doi.org/10.1016/j.adolescence.2010.09.003.

Coyne, L. W., McHugh, L., \& Martinez, E. R. (2011a). Acceptance and commitment therapy (ACT): Advances and applications with children, adolescents, and families. Child and Adolescent Psychiatric Clinics of North America, 20(2), 379-399.

Coyne, L. W., McHugh, L., \& Martinez, E. R. (2011b). Acceptance and commitment therapy (ACT): advances and applications with children, adolescents, and families. Child and Adolescent Psychiatric Clinics of North America, 20(2), 379-399. https://doi.org/10.1016/j.chc.2011.01.010.

Cross, W., West, J., Wyman, P. A., Schmeelk-Cone, K., Xia, Y., Tu, X., et al. (2015). Observational measures of implementer fidelity for a school-based preventive intervention: Development, reliability, and validity. Prevention Science, 16(1), 122-132. https://doi.org/10.1007/s11121-014-0488-9.

Dunst, C. J., Trivette, C. M., \& Raab, M. (2013). An implementation science framework for conceptualizing and operationalizing fidelity in early childhood intervention studies. Journal of Early Intervention, 35(2), 85-101. https://doi.org/10.1177/1053815113502235.

Durlak, J. A., \& DuPre, E. P. (2008). Implementation matters: A review of research on the influence of implementation on program outcomes and the factors affecting implementation. American Journal of Community Psychology, 41(3-4), 327-350. https://doi.org/10.1007/s10464-008-9165-0. 
Ernst, M. M., \& Mellon, M. W. (2016). Acceptance and Commitment Therapy (ACT) to foster resilience in pediatric chronic illness. In Child and adolescent resilience within medical contexts (pp. 193-207).

Fardell, J. E., Wakefield, C. E., Patterson, P., Lum, A., Cohn, R. J., Pini, S. A., et al. (2018). Narrative review of the educational, vocational, and financial needs of adolescents and young adults with cancer: Recommendations for support and research. Journal of Adolescent and Young Adult Oncology, 7(2), 143-147. https://doi.org/10.1089/jayao.2017.0086.

Friend, A. J., Feltbower, R. G., Hughes, E. J., Dye, K. P., \& Glaser, A. W. (2018). Mental health of longterm survivors of childhood and young adult cancer: A systematic review. International Journal of Cancer. https://doi.org/10.1002/ijc.31337.

GBD 2017 Childhood Cancer Collaborators. (2019). The global burden of childhood and adolescent cancer in 2017: An analysis of the Global Burden of Disease Study 2017. The Lancet Oncology, 20(9), 1211-1225. https://doi.org/10.1016/S1470-2045(19)30339-0

Gunst, D. C., Kaatsch, P., \& Goldbeck, L. (2016). Seeing the good in the bad: Which factors are associated with posttraumatic growth in long-term survivors of adolescent cancer? Supportive Care in Cancer, 24(11), 4607-4615. https://doi.org/10.1007/s00520-016-3303-2.

Halliburton, A. E., \& Cooper, L. D. (2015). Applications and adaptations of Acceptance and Commitment Therapy (ACT) for adolescents. Journal of Contextual Behavioral Science, 4(1), 1-11. https:// doi.org/10.1016/j.jcbs.2015.01.002.

Hayes, S. C., Luoma, J. B., Bond, F. W., Masuda, A., \& Lillis, J. (2006). Acceptance and commitment therapy: Model, processes and outcomes. Behaviour Research and Therapy, 44(1), 1-25. https:// doi.org/10.1016/j.brat.2005.06.006.

Hayes, S. C., Strosahl, K. D., \& Wilson, K. G. (Eds.). (1999). Acceptance and commitment therapy: An experiential approach to behavior change. New York: The Guildford Press.

Ing, V., Patterson, P., Szabo, M., \& Allison, K. R. (2019). Interventions available to adolescents and young adults bereaved by familial cancer: A systematic literature review. BMJ Supportive and Palliative Care. https://doi.org/10.1136/bmjspcare-2019-001959.

Janin, M. M. H., Ellis, S. J., Wakefield, C. E., \& Fardell, J. E. (2018). Talking about cancer among adolescent and young adult cancer patients and survivors: A systematic review. Journal of Adolescent and Young Adult Oncology. https://doi.org/10.1089/jayao.2017.0131.

Jones, P., Blunda, M., Biegel, G., Carlson, L. E., Biel, M., \& Wiener, L. (2013). Can mindfulnessbased interventions help adolescents with cancer? Psychooncology, 22(9), 2148-2151. https://doi. org/10.1002/pon.3251.

Kendall, P. C., Gosch, E., Furr, J. M., \& Sood, E. (2008). Flexibility within fidelity. Journal of the American Academy of Child and Adolescent Psychiatry, 47(9), 987-993. https://doi.org/10.1097/ CHI.0b013e31817eed2f.

Kim, B., White, K., \& Patterson, P. (2016). Understanding the experiences of adolescents and young adults with cancer: A meta-synthesis. European Journal of Oncology Nursing, 24, 39-53. https:// doi.org/10.1016/j.ejon.2016.06.002.

Livheim, F., Hayes, L., Ghaderi, A., Magnusdottir, T., Högfeldt, A., Rowse, J., et al. (2014). The effectiveness of acceptance and commitment therapy for adolescent mental health: Swedish and australian pilot outcomes. Journal of Child and Family Studies, 24(4), 1016-1030. https://doi. org/10.1007/s10826-014-9912-9.

Martins, A., Taylor, R. M., Morgan, S., \& Fern, L. A. (2017). Being normal, not vulnerable: case study of a 2-day residential programme for young adults with cancer. British Medical Journal Open, 7(7), e015309. https://doi.org/10.1136/bmjopen-2016-015309.

McDonnell, G. A., Salley, C. G., Barnett, M., DeRosa, A. P., Werk, R. S., Hourani, A., et al. (2017). Anxiety among adolescent survivors of pediatric cancer. Journal of Adolescent Health, 61(4), 409423. https://doi.org/10.1016/j.jadohealth.2017.04.004.

Mendel, P., Meredith, L. S., Schoenbaum, M., Sherbourne, C. D., \& Wells, K. B. (2008). Interventions in organizational and community context: A framework for building evidence on dissemination and implementation in health services research. Administration and Policy In Mental Health, 35(1-2), 21-37. https://doi.org/10.1007/s10488-007-0144-9.

Niepage, M., Georgievski, G., Shama, W., \& Lucchetta, S. (2018). Exploring adolescents' cancer journey through photovoice: A narrative synthesis. Journal of Adolescent and Young Adult Oncology, 7(1), 15-21. https://doi.org/10.1089/jayao.2017.0073.

Patterson, P., \& McDonald, F. E. (2015). "Being Mindful": Does it help adolescents and young adults who have completed cancer treatment? Journal of Pediatric Oncology Nursing, 32(4), 189-194. https://doi.org/10.1177/1043454214563401. 
Patterson, P., McDonald, F. E., Ciarrochi, J., Hayes, L., Tracey, D., Wakefield, C. E., et al. (2015). A study protocol for truce: A pragmatic controlled trial of a seven-week acceptance and commitment therapy program for young people who have a parent with cancer. BMC Psychology, 3, 31. https://doi. org/10.1186/s40359-015-0087-y.

Penn, A., \& Kuperberg, A. (2018). Psychosocial support in adolescents and young adults with cancer. The Cancer Journal, 24(6), 321-327.

Perez, D., Van der Stuyft, P., Zabala, M. C., Castro, M., \& Lefevre, P. (2016). A modified theoretical framework to assess implementation fidelity of adaptive public health interventions. Implementation Science, 11(1), 91. https://doi.org/10.1186/s13012-016-0457-8.

Pielech, M., Vowles, K. E., \& Wicksell, R. (2017). Acceptance and commitment therapy for pediatric chronic pain: Theory and application. Children (Basel). https://doi.org/10.3390/children4020010.

Pini, S., Hugh-Jones, S., Shearsmith, L., \& Gardner, P. (2019). 'What are you crying for? I don't even know you': The experiences of teenagers communicating with their peers when returning to school. European Journal of Oncology Nursing, 39, 28-34. https://doi.org/10.1016/j.ejon.2018.12.010.

Rourke, M. T., Samson, K. K., \& Kazak, A. E. (2015). Psychological aspects of long-term survivorship. In C. L. Schwartz, W. L. Hobbie, L. S. Constine, \& K. S. Ruccione (Eds.), Survivors of childhood and adolescent cancer: A multidisciplinary approach. Heidelberg: Springer.

Sansom-Daly, U. M., Wakefield, C. E., Bryant, R. A., Butow, P., Sawyer, S., Patterson, P., et al. (2012). Online group-based cognitive-behavioural therapy for adolescents and young adults after cancer treatment: A multicenter randomised controlled trial of Recapture Life-AYA. BMC Cancer, 12, 339. https ://doi.org/10.1186/1471-2407-12-339.

Sansom-Daly, U. M., Wakefield, C. E., Bryant, R. A., Patterson, P., Anazodo, A., Butow, P., et al. (2019). Feasibility, acceptability, and safety of the Recapture Life videoconferencing intervention for adolescent and young adult cancer survivors. Psycho-Oncology, 28(2), 284-292.

Song, H., Nam, Y., Gould, J., Sanders, W. S., McLaughlin, M., Fulk, J., et al. (2012). Cancer survivor identity shared in a social media intervention. Journal of Pediatric Oncology Nursing, 29(2), 80-91. https ://doi.org/10.1177/1043454212438964.

Steinberg, L., \& Morris, A. S. (2001). Adolescent development. Annual Review of Psychology, 52.

Swain, J., Hancock, K., Dixon, A., \& Bowman, J. (2015). Acceptance and Commitment Therapy for children: A systematic review of intervention studies. Journal of Contextual Behavioral Science, 4(2), 73-85.

Tsangaris, E., Johnson, J., Taylor, R., Fern, L., Bryant-Lukosius, D., Barr, R., et al. (2014). Identifying the supportive care needs of adolescent and young adult survivors of cancer: A qualitative analysis and systematic literature review. Supportive Care in Cancer, 22(4), 947-959. https://doi.org/10.1007/s0052 0-013-2053-7.

Wakefield, C. E., McLoone, J., Butow, P., Lenthen, K., \& Cohn, R. J. (2013). Support after the completion of cancer treatment: Perspectives of Australian adolescents and their families. European Journal of Cancer Care, 22(4), 530-539.

Walker, E., Martins, A., Aldiss, S., Gibson, F., \& Taylor, R. M. (2016). Psychosocial interventions for adolescents and young adults diagnosed with cancer during adolescence: A critical review. Journal of Adolescent and Young Adult Oncology, 5(4), 310-321. https://doi.org/10.1089/jayao.2016.0025.

Woodgate, R. L. (2005). A different way of being: Adolescents' experiences with cancer. Cancer Nursing, $28(1), 8-15$.

Yi, J., Zebrack, B., Kim, M. A., \& Cousino, M. (2015). Posttraumatic growth outcomes and their correlates among young adult survivors of childhood cancer. Journal of Pediatric Psychology, 40(9), 981-991. https://doi.org/10.1093/jpepsy/jsv075.

Publisher's Note Springer Nature remains neutral with regard to jurisdictional claims in published maps and institutional affiliations. 


\section{Affiliations}

Kristina Clarke ${ }^{1}$ Pandora Patterson ${ }^{1,2}\left(\mathbb{D}\right.$. Fiona E. J. McDonald ${ }^{1,2}(1) \cdot$

Claire E. Wakefield ${ }^{3,4}\left(\right.$ ) Ursula Sansom-Daly ${ }^{3,4} @$ Brad Zebrack $^{5}$

1 CanTeen Australia, GPO Box 3821, Sydney, NSW 2001, Australia

2 Cancer Nursing Research Unit, The University of Sydney, Sydney, Australia

3 School of Women's \& Children's Health, UNSW, Sydney, Australia

4 Kids Cancer Centre, Sydney Children's Hospital, Sydney, Australia

5 School of Social Work, The University of Michigan, Ann Arbor, MI, USA 